Italy looks to next supernova

\section{Mont Blanc}

FOR Oscar Saavedra and his colleagues at the Italian national research council (CNR) Istituto di Cosmogeofisica in Turin and the Soviet Academy of Sciences, the 1987 supernova in the Large Magellanic Cloud came eight years too early.

Four years ago, the Italian/Soviet collaboration was the first to report a burst of neutrinos from SN1987A, which they had detected at their underground observatory at Mont Blanc, 2,000 metres below the Italian/ French border in an emergency bay off the Mont Blanc road tunnel. That neutrino sighting, however, is now thought by most physicists to have probably been due to background noise and the detectors apparently missed a real neutrino burst that occurred several hours later.

So now INFN, the Italian national institute for nuclear physics, is building a larger neutrino detector at the Gran Sasso underground physics complex about $650 \mathrm{~km}$ southeast of Mont Blanc. This Large Volume Detector (LVD), which is expected to be completed in three to four years, might well have helped to establish whether the Mont Blanc detection was a real neutrino event linked to SN1987A if the LVD had existed at the time, Saavedra says.

Standard astrophysical theory holds that a collapsing star, before it explodes as a supernova, should release a burst of neutrinos, and the Mont Blanc team, headed by Carlo Castagnoli, believed that they had discovered such a burst. They were the first to report neutrino detection after other astronomers had reported optical observation of the supernova on 24 February 1987. But four and a half hours after the Mont Blanc burst, which consisted of five events over several seconds, a second burst was recorded independently by the Japanese detector at the Kamioka zinc mine and the US Irvine-Michigan-Brookhaven (IMB) device, located in an Ohio salt mine.

Physicists disagree on what the detection of two distinct signals implies. Because theory predicts only a single neutrino burst from a collapsing star, and because the IMB and Kamioka observations fitted well with theoretical predictions, the general perception among astrophysicists is that the Mont Blanc burst was background noise, most probably caused by penetrating radiation from the surrounding rock. Such a burst would be expected about once every three years simply from random fluctuations.

Saavedra believes it is possible that both the Mont Blanc detection and the later bursts recorded simultaneously in the United States and Japan could have been genuine events linked to SN1987A. This would require the star to have collapsed initially to a neutron star, releasing low-energy neutrinos picked up at Mont Blanc, but below the energy threshold of the IMB and Kamioka devices.
A second collapse to a black hole would then explain the neutrino burst recorded by IMB and Kamioka. At Mont Blanc, this burst may have been indistinguishable from the background noise.

Whatever happened, the combination of the Mont Blanc neutrino detector and the Gran Sasso LVD now under construction should give astrophysicists a clearer picture of the next nearby supernova.

Like the Mont Blanc device, the LVD will be a liquid-scintillation detector. The Mont Blanc detector contains 90 tonnes of an organic solvent held in 72 separate $1.5 \mathrm{~m}^{3}$ tanks. The solvent fluoresces when neutrinos, cosmic muons or other particles pass through it, and sensitive photomultipliers

\section{Turin}

EuROPE should aim to take the lead in oceanographic research in the $1990 \mathrm{~s}$, says Umberto Colombo, president of the European Science Foundation (ESF), summing up last week's meeting in Turin on Oceans, Climate and Man, organized by the Foundation San Paolo.

The difficulties are several, including finding funds for oceanographic studies and turning out enough trained scientists, but the rewards would be worth the effort. Given the rapid expansion of oceanographic data and improvements in supercomputing expected over the next decade, many delegates at the Turin meeting argued that it may soon be possible to provide operational forecasts of the behaviour of the oceans, similar to the atmospheric forecasts used in medium-range weather prediction.

Ocean forecasts would provide information useful for shipping, fisheries, pollution control and coastal protection.

Added to this are the spin-offs for climate prediction: the difficulty of including the behaviour of the oceans in general circulation climate models is one of the most serious constraints on the reliable prediction of climate change.

The obvious model for a European ocean forecasting centre is the European Centre for Medium-Range Weather Forecasting (ECMWF), at Reading, England. Since 1979, ECMWF has been providing medium-range (three- to ten-day) weather forecasts for the meteorological agencies of its 18 subscriber countries, and has been a "roaring success", according to John Woods, director of marine and atmospheric sciences at the UK Natural Environment Research Council. But a parallel centre for ocean forecasting is still several years off, he says. The idea is one of many being considered by the European Committee on Ocean and Polar Science, a joint initiative of the European around the tanks detect the emitted light, signalling the passage of the particle. The LVD is planned to be a scaled-up version of the Mont Blanc detector.

Some workers in the field say, however, that the Italians would have been better advised to abandon liquid-scintillation technology for their next detector. With a bank of relatively large scintillation tanks such as those planned for Gran Sasso, it is difficult to track the progress of particles through the detector. On the other hand, the devices at IMB and Kamioka, which work by detecting the Cerenkov radiation given off by particles passing through water, do allow researchers to follow the tracks of particles. This makes it easier to distinguish between different types of particles, and to tell if some events are simply due to faults in the equipment.

Peter Aldhous

\title{
Europe in the vanguard?
}

Communities (EC) and the European Science Foundation (ESF), to present to ministers from European governments in 1994.

Nadia Pinardi, from the Italian national research council (CNR) Istituto per lo Studio delle Metodologie Geofisiche Ambientali at Modena, says that there is now enough data collected every 15 days to feed into an ocean forecasting model. The resulting forecasts would be coarser than those produced by meteorologists (ECMWF bases its forecasts on the daily collation of atmospheric data), but would be a useful start, she says.

Pinari also wants the EC's research programmes to provide grants for European scientists to take part in the World Ocean Circulation Experiment (WOCE) - the huge international oceanography project planned to run until 1997 as part of the World Climate Research Programme - and for European researchers to take part in processing the large volume of oceanographic data expected from the European Space Agency's ERS-1 remote-sensing satellite, due for launch next month.

Although the EC's marine science and technology (MAST) programme has been valuable in stimulating European oceanography, Pinadi says, the EC has so far not paid for WOCE research. This is not so much a problem in countries such as Britain and Germany, where there are national programmes of WOCE research. But in most southern European countries, where national governments have shown little interest in WOCE, EC funding is the only hope for oceanographers who wish to participate in the largest research project in their field.

The biggest single obstacle for the progress of oceanography in Europe is undertraining, says Woods. In Britain, for example, only ten per cent of scientists entering the field have previous specialist training in oceanography

Peter Aldhous 